\title{
3D microvessel-mimicking ultrasound phantoms produced with a scanning motion system
}

\author{
Ryan C. Gessner, Roshni Kothadia, Steven Feingold, and Paul A. Dayton
}

\section{Abstract}

Ultrasound techniques are currently being developed which can assess the vascularization of tissue as a marker for therapeutic response. Some of these ultrasound imaging techniques seek to extract quantitative features about vessel networks, while high-frequency imaging also allows individual vessels to be resolved. The development of these new techniques, and subsequent imaging analysis strategies, necessitates an understanding of their sensitivities to vessel and vessel network structural abnormalities. Constructing in-vitro flow phantoms for this purpose can be prohibitively challenging, as simulating precise flow environments with non-trivial structures is often impossible using conventional methods of construction for flow phantoms. Presented in this manuscript is a method to create predefined structures with $<10 \mu \mathrm{m}$ precision using a three-axis motion system. The application of this technique is demonstrated for the creation of individual vessel and vessel networks, which can easily be made to simulate the development of structural abnormalities typical of diseased vasculature in-vivo. Additionally, beyond facilitating the creation of phantoms which would be otherwise very challenging to construct, the method presented herein enables one to precisely simulate very slow blood flow, respiration artifacts, and to measure imaging resolution.

\section{Keywords}

phantom; microbubble; contrast; 3D imaging; vessel; vessel network; vessel architecture

\section{Introduction}

When assessing disease response to therapy, the relationship between vascularization and disease state is significant. Indeed, many new imaging techniques are being developed to detect subtle changes in vascularization as a metric for quantifying the effects of administered therapies (Schaefer et al. 2010, Pollard and Larson 2010, Hwang et al. 2010). Ultrasound is a popular imaging modality for preclinical and clinical disease characterization alike, as it is portable, safe, and relatively inexpensive. There have been many studies recently published which implement ultrasound, or demonstrate developments to ultrasound imaging techniques for monitoring disease state by way of blood flow characterization. Some of these strategies seek to quantify the amount of vascularization within the tissue by using Doppler techniques (Pinter and Lacefield 2010, Wang et al. 2010, Alcázar et al. 2010, Palmowski et al. 2008), or contrast image-subtraction approaches

(C) 2010 World Federation for Ultrasound in Medicine and Biology. Published by Elsevier Inc. All rights reserved. Corresponding author: Paul A. Dayton, Address: 304 Taylor Hall, 109 Mason Farm Road, Chapel Hill, NC, 27599-6136, Phone: (919) - 843-9521, Fax: (919)-843-9520, padayton@bme.unc.edu.

Publisher's Disclaimer: This is a PDF file of an unedited manuscript that has been accepted for publication. As a service to our customers we are providing this early version of the manuscript. The manuscript will undergo copyediting, typesetting, and review of the resulting proof before it is published in its final citable form. Please note that during the production process errors may be discovered which could affect the content, and all legal disclaimers that apply to the journal pertain. 
(Loveless et al. 2008, Ding et al. 2008). Other strategies attempt to extract information about the rates at which blood flows through the tissue using contrast-based destructionreperfusion (or "flash replenishment") techniques (Wei et al. 1998, Pollard et al. 2002, Metoki et al. 2006, Chen et al. 2006, Shiraishi et al. 2008, Pollard et al. 2009, Feingold et al. 2010). Additionally, others have attempted to extract quantifiable metrics for vessel and vessel network structure as a means to understand disease progression (Sugimoto et al. 2010, Molinari et al. 2010, Shiraishi et al. 2008, Huang et al. 2008). Of particular interest is vessel tortuosity, which is a morphological abnormality exhibited by cancerous vasculature in both humans and rodents ( $\mathrm{Li}$ et al. 2000, Jain 2005), and has been used as a marker for disease state and therapeutic response in clinical studies based on image-derived data (Bullitt et al. 2004, Bullitt et al. 2007).

The development and implementation of ultrasonic vascular imaging techniques, along with their corresponding image analysis strategies, necessitates an understanding of their resolution and detection sensitivity as well as their sensitivity to corruption by the unfortunate realities of in-vivo imaging studies, such as respiratory motion and signal attenuation. In-vitro phantoms are utilized as a means to simulate anatomically relevant structures in a controlled environment to test sensitivity as well as the effects of these variables. Blood vessel mimicking flow phantoms are often constructed of either acoustically transparent cellulose tubes set in gelatin for support, or wall-less gelatin bores through which contrast agents can be flowed and imaged. While these techniques are sufficient to test an imaging strategy's sensitivity or signal to noise ratio, they are suboptimal in creating environments for simulating blood vessel networks which have nontrivial structural details. Furthermore, attempts to simulate structural features in the hundredmicron range can be prohibitively difficult simply due to the challenge of working with materials on a sub-millimeter scale. This is a problem which most affects those seeking to characterize the structural morphologies of individual vessels or vessel networks as markers for disease.

Presented in this manuscript is a novel method of generating individual 3D vessel phantoms with predefined coordinates of nearly any morphology. Our microvessel phantoms can be constructed with $<10 \mu \mathrm{m}$ precision. The technique is extended to the generation of entire vasculature networks via basic image addition of the independently constructed vessels. The principle behind our strategy is that an object is continuously imaged while also being translated in a controlled manner by a computer-controlled motion stage. If this motion occurs between imaging frames, it will appear smooth and continuous in the final 3D image. The strategy could be beneficial for determining the accuracy and sensitivity of imaging and image-analysis techniques seeking to quantify the structural morphology of vessels or vessel networks, as well techniques quantifying blood flow speed. It can also assist in determining imaging resolution, sensitivity to artifacts, and detection sensitivity for any of the ultrasound imaging strategies commonly implemented for vascularity assessment.

\section{Materials and methods}

\section{Microcapillary and contrast agents}

The complex microvessel flow phantoms generated in this manuscript were created by spatially manipulating a contrast-filled microcapillary during imaging. The microcapillary was a single thin-walled $200 \mu \mathrm{m}$ cellulose microtube (Spectrum Labs - Rancho Dominguez, CA) coupled with hot-melt glue to $380 \mu \mathrm{m}$ polyethylene tubes (Becton Dickinson, Sparks, $\mathrm{MD}$ ). The cellulose tube region was imaged, while the larger polyethylene tubes were implemented to facilitate coupling with the syringe for contrast injection. A polydisperse distribution of microbubble contrast agents (mean diameter $0.9 \mu \mathrm{m}$, standard deviation 0.45 $\mu \mathrm{m}$ ) were prepared as previously described (Streeter et al. 2009) and pumped through the 
tubes via coupling with a 27 -gauge blunt needle. The tube was suspended in a water bath from one of the arms of a precision three-axis motion stage (Newport model XPS - Irvine, CA). A contrast-specific high resolution imaging method was implemented on a Vevo 770 ultrasound system (VisualSonics, Toronto CA), as previously described (Gessner et al. 2010).

\section{Motion system}

A custom LabView (National Instruments - Austin, TX) program was written on a personal computer (PC) to control the motion stage, as seen in Figure 1A. The transducer was positioned above the tube, with the tube's axis oriented normal to the imaging plane, and parallel to the direction of the transducer's translational motor stage as seen in Figure 1B. The frame trigger from the ultrasound system was used to trigger the PC, which then activated the motion stage to move the cellulose tube to the next X-Y coordinate in its centerline. The imaging system was set to acquire images at a fixed rate of $2 \mathrm{~Hz}$, and the motion stage would increment the position of the microtube once between each image frame acquisition. 3D images were acquired via the ultrasound system's stepwise translational motor stage, with an inter-frame step size of $0.05 \mathrm{~mm}$. This yielded nearly isotropic voxels in the resulting 3D images.

\section{Image acquisition}

Prior to phantom imaging, $\mathrm{X}-\mathrm{Y}-\mathrm{Z}$ coordinates of the desired vessel's centerline were generated in Matlab (The Mathworks - Natick, MA) and exported as tab-delimited text files. For the remainder of this manuscript, the $\mathrm{X}, \mathrm{Y}$, and $\mathrm{Z}$ axes correspond to the transducer's lateral, axial, and elevational directions respectively. Any arbitrarily-shaped vessel curve could be generated, provided the resulting centerline did not fail the "vertical line test" in the $\mathrm{X}-\mathrm{Y}$ plane. This caveat was due to the method by which we were acquiring our 3D images: the transducer only would acquire a single image at every $\mathrm{Z}$ location, thus it was impossible for a vessel to loop back on itself in the $\mathrm{Z}$ direction, although this could be done with modification of the system.

Single vessel phantoms were generated and imaged with different morphologies, including helical-shaped and meandering vessels with random tortuosity added. Additionally, by summing up images of single vessel phantoms, we were able to create a multiple vessel composite phantom to simulate a vessel network. Our methodology for 3D single vessel phantom generation is illustrated by the cartoon diagram in Figure 2. The transducer's Z position is in red, and is controlled by the ultrasound system. In these examples, as the probe is scanned the Newport motion stage moves the tube A) up and down, or B) in a circle within the $2 \mathrm{D}$ ultrasound imaging plane. The result is a sinusoid or a helix in the resulting 3D ultrasound image.

In order to use this system to assess contrast intensity as a function of axial depth and pulsing pressure, coordinates were generated for a simple linear and diagonal phantom originating deep in the field of view and concluding very close to the transducer. The gradual slope of this phantom enabled a precise characterization of contrast sensitivity as a function of depth, in $100 \mu \mathrm{m}$ intervals. Moreover, by modulating the pressure output of the transducer, we characterized contrast sensitivity for depth of field over multiple pulsing pressures between 300 and $650 \mathrm{kPa}$. 


\section{Results}

\section{D tortuosity vessel phantom generation}

We discuss specifically the generation of two types of vessel phantoms of potential interest to those studying vessel structure and morphology. The first, seen in Figure 3, is a single vessel which develops tortuosity abnormalities along its path. In this study, a single gently meandering vessel, representing "healthy" vasculature has noise randomly added to the X and $\mathrm{Y}$ coordinate of each point along its centerline. The amplitude of the noise is modulated to create a more tortuous vessel. The most tortuous vessel has a mean deviation of $445 \mu \mathrm{m}$ from the healthy vessel's centerline. This type of phantom could be utilized to simulate morphological abnormalities relevant to tumor response to treatment, as described by Bullitt et al (2004).

\section{Bifurcating vessel phantom generation}

Another type of phantom generated was a composite-vessel structure composed of multiple independent vessel phantoms. First, X-Y-Z coordinates were independently generated for each of the four vessels in Matlab, and the resulting four 3D images of each vessel added together to simulate a single continuous vessel network (Figures 4A-C). The 3D ultrasound image in Figure 4D was rendered in ImageJ (National Institutes of Health - Bethesda, MD). This vessel network was designed to have three different bifurcations. At each bifurcation, the two daughter vessels diverge in opposite directions. Prior to a bifurcation, the daughter vessels resulting from that bifurcation trace equivalent paths to ensure smoothness at these junctions. By varying the angles at which daughter vessels diverge, one could simulate vascular changes due to the onset of disease. The angles at which vessels diverge have been linked to the presence of liver disease (Sugimoto et al. 2010), with the fibrous tissue in diseased livers causing wider divergence angles between hepatic vessels. Thus this type of phantom could be used to measure an imaging system's sensitivity to these types of morphological changes occurring in in-vivo vasculature.

\section{Measuring contrast sensitivity}

By simulating a diagonal phantom oriented in the $\mathrm{Y}-\mathrm{Z}$ plane, we were able to measure our contrast detection sensitivity as a function of imaging pressure and location within the field of view. The tube was raised in $100 \mu \mathrm{m}$ increments between images, and was imaged at points within $6 \mathrm{~mm}$ from the acoustic focus. Images were acquired at eight different pulsing pressures between 300 and $650 \mathrm{kPa}$. The data is summarized in Figure 5A, with a corresponding cartoon demonstrating the orientation of the transducer and tube in Figure 5B.

The detection sensitivity in Figure 5A was quantified by averaging the pixels with image intensities above the noise floor in each 2D image in the 3D dataset. The phantom tube's mean intensities in each 2D image could be directly mapped to the distance from the tube to the transducer's focus since this relationship was governed by the coordinates used by the motion stage to create the phantom.

\section{Discussion and Conclusions}

\section{Advantages of the phantom generation technique}

The most enabling component of the technique described in this manuscript for 3D flow phantom generation is the ability to create precise structures in the ultrasound data that would otherwise be prohibitively difficult or impossible to construct using conventional flow phantom materials. We have demonstrated the capability to generate phantoms with shapes such as helices, sinusoids, vessels with varying degress of tortuoisity, and bifurcating vessels; all with effective diameters on the order of 200 microns. Additionally, the micron- 
level accuracy of today's computer controlled motion stages offers fine control over phantom precision.

There exist other applications of this phantom generation technique which were not tested in these studies. For example, the motion stage-tube apparatus could be used to determine the sensitivity to slowly flowing blood by modulating the speed of the motion stage and measuring the reported flow speed against the known speed of the tube. One could also use the setup to simulate respiration artifacts of different magnitudes in order to determine an imaging strategy's sensitivity to this reality of in-vivo imaging studies. Finally, the approach could be used to measure an imaging strategy's resolution. If two tubes are simulated which pass through each other at a shallow angle of attack, the actual separation distance between the tubes' centerlines is known, so one could extract the last point at which they appear distinct and know the analogous physical separation between them (i.e. the imaging system's spatial resolution).

\section{Limitations of the phantom generation technique}

While the phantom generation technique presented herein is a novel means to more accurately construct micro-flow phantoms for ultrasound imaging studies, there are several limitations of the technique worth noting. First and foremost, it must be implemented in a water bath, as the tube needs a smooth acoustically transparent medium through which to move. This then makes it necessary to add speckle noise as a post-processing step if one is interested in analyzing vessels in a more realistic imaging context. Since the process of building up vessel networks presented in this manuscript is via simple voxel addition, the signal to noise ratio (SNR) between the vessels and the background noise in the final composite vessel images is spatially variant as a function of how many vessel signals are added together at a given location. The noise power increases uniformly throughout the image volume of the composite vessel phantom as each individual vessel phantom is added to the composite image; the signal power within the vessels does not always increase at all locations, however. In some locations of a composite image multiple vessels can be present, such as in the region before a bifurcation, and thus when they are added the final "vessel signal" increases $\mathrm{N}$ times (where $\mathrm{N}$ is the number of vessels present at a location). After vessels bifurcate and trace unique trajectories through the image space, their signals do not add any longer and thus are reduced relative to the increased noise power. While this was not accounted for in these preliminary proof-of-concept imaging studies, one could compensate for this by characterizing the noise of the imaging system and normalizing the noise to the vessel signals in a post-processing step after the composite vessel networks had been constructed, as the centerlines of each vessel within the network are known.

An additional limitation of the phantom generation technique is the amount of translation which can be used while still maintaining the illusion of a smooth and continuous tube through 3D space. Because the tube was oriented normal to the imaging plane, every 2D image has a nearly circular cut through the tube. If phantoms with steep angles relative to the plane of the image are created, the circular tube will need to translate many pixels between imaging frames and thus have a stratified appearance in the final 3D image. This effect can be partially accounted for by choosing small elevational step sizes. Although it was not necessary to consider the orientation of the tube relative to the imaging plane with our contrast detection strategy, those implementing Doppler techniques for contrast detection would need to take this into consideration to avoid the detection sensitivity issues associated with 90 degree Doppler angles. Additionally, if one is interested in achieving non-trivial velocity streamlines within a vessel phantom generated by the method presented herein, additional degrees of freedom must be added to their experimental apparatus. For instance, if one wanted to simulate a change in flow direction at a bifurcation they would need to rotate the transducer relative to the tube at the appropriate location along the vessel's 
centerline (or equivalently: rotate the tube beneath the transducer). This would become increasingly difficult in situations where a vessel's trajectory varied in both the $\mathrm{X}$ and $\mathrm{Y}$ dimensions, such as those in Figure 4, as the transducer would need to undergo two independent rotations. While not impossible, it is likely that this type of imaging procedure would be prohibitively difficult. Thus, we propose this phantom generation method as primarily of interest for those interested in assessing complex vessel morphology.

\section{Acknowledgments}

The authors appreciate the assistance of Marc Lukacs for offering his technical assistance with synchronizing the Vevo 770 ultrasound system to LabView. Funding for this research was supported by the NIH through EB009066 and through a pilot study grant from the UNC Lineberger Cancer Center. R.C.G. was supported in part by the Graduate Research Fellowship Program of the National Science Foundation.

\section{References}

Alcázar JL, Jurado M, López-García G. Tumor vascularization in cervical cancer by 3-dimensional power Doppler angiography: correlation with tumor characteristics. Int J Gynecol Cancer. 2010; 20:393-397. [PubMed: 20375803]

Bullitt E, Ewend MG, Aylward S, Lin W, Gerig G, Joshi S, Jung I, Muller K, Smith JK. Abnormal vessel tortuosity as a marker of treatment response of malignant gliomas: preliminary report. Technol Cancer Res Treat. 2004; 3:577-584. [PubMed: 15560715]

Bullitt E, Lin NU, Smith JK, Zeng D, Winer EP, Carey LA, Lin W, Ewend MG. Blood vessel morphologic changes depicted with MR angiography during treatment of brain metastases: a feasibility study. Radiology. 2007; 245:824-830. [PubMed: 17954616]

Chen NG, Fowlkes JB, Carson PL, LeCarpentier GL. Rapid 3D imaging of contrast flow: demonstration of a dual beam technique. Ultrasound Med Biol. 2007; 33:915-923. [PubMed: 17466444]

Ding Y, Boguslawski EA, Berghuis BD, Young JJ, Zhang Z, Hardy K, Furge K, Kort E, Frankel AE, Hay RV, Resau JH, Duesbery NS. Mitogen-activated protein kinase kinase signaling promotes growth and vascularization of fibrosarcoma. Mol Cancer Ther. 2008; 7:648-658. [PubMed: 18319331]

Feingold S, Gessner R, Guracar IM, Dayton PA. Quantitative volumetric perfusion mapping of the microvasculature using contrast ultrasound. Invest Radiol. 2010; 45:669-674. [PubMed: 20808232]

Gessner R, Lukacs M, Lee M, Cherin E, Foster FS, Dayton PA. High-resolution, high-contrast ultrasound imaging using a prototype dual-frequency transducer: in vitro and in vivo studies. IEEE Trans Ultrason Ferroelectr Freq Control. 2010; 57:1772-1781. [PubMed: 20679006]

Huang SF, Chang RF, Moon WK, Lee YH, Chen DR, Suri JS. Analysis of tumor vascularity using three-dimensional power Doppler ultrasound images. IEEE Trans Med Imaging. 2008; 27:320-330. [PubMed: 18334428]

Hwang M, Hariri G, Lyshchik A, Hallahan DE, Fleischer AC. Correlation of quantified contrastenhanced sonography with in vivo tumor response. J Ultrasound Med. 2010; 29:597-607. [PubMed: 20375378]

Jain RK. Normalization of tumor vasculature: an emerging concept in antiangiogenic therapy. Science. 2005; 307:58-62. [PubMed: 15637262]

Li CY, Shan S, Huang Q, Braun RD, Lanzen J, Hu K, Lin P, Dewhirst MW. Initial stages of tumor cell-induced angiogenesis: evaluation via skin window chambers in rodent models. J Natl Cancer Inst. 2000; 92:143-147. [PubMed: 10639516]

Loveless ME, Li X, Huamani J, Lyshchik A, Dawant B, Hallahan D, Gore JC, Yankeelov TE. A method for assessing the microvasculature in a murine tumor model using contrast-enhanced ultrasonography. J Ultrasound Med. 2008:271699-271709.

Metoki R, Moriyasu F, Kamiyama N, Sugimoto K, Iijima H, Xu HX, Aoki T, Miyata Y, Yamamoto K, Kudo K, Shimizu M, Yamada M. Quantification of hepatic parenchymal blood flow by contrast ultrasonography with flash-replenishment imaging. Ultrasound Med Biol. 2006; 32:1459-1466. [PubMed: 17045864] 
Molinari F, Mantovani A, Deandrea M, Limone P, Garberoglio R, Suri JS. Characterization of single thyroid nodules by contrast-enhanced 3-D ultrasound. Ultrasound Med Biol. 2010; 36:1616-1625. [PubMed: 20800947]

Palmowski M, Huppert J, Hauff P, Reinhardt M, Schreiner K, Socher MA, Hallscheidt P, Kauffmann GW, Semmler W, Kiessling F. Vessel fractions in tumor xenografts depicted by flow- or contrastsensitive three-dimensional high-frequency Doppler ultrasound respond differently to antiangiogenic treatment. Cancer Res. 2008; 68:7042-7049. [PubMed: 18757418]

Pinter SZ, Lacefield JC. Objective selection of high-frequency power Doppler wall filter cutoff velocity for regions of interest containing multiple small vessels. IEEE Trans Med Imaging. 2010; 29:1124-1139. [PubMed: 20236878]

Pollard RE, Sadlowski AR, Bloch SH, Murray L, Wisner ER, Griffey S, Ferrara KW. Contrast-assisted destruction-replenishment ultrasound for the assessment of tumor microvasculature in a rat model. Technol Cancer Res Treat. 2002; 1(6):459-70. [PubMed: 12625773]

Pollard RE, Dayton PA, Watson KD, Hu X, Guracar IM, Ferrara KW. Motion corrected cadence CPS ultrasound for quantifying response to vasoactive drugs in a rat kidney model. Urology. 2009; 74(3):675-81. [PubMed: 19589583]

Pollard RE, Larson RF. Dynamic contrast-enhanced computed tomography for the quantification of tumor response to vasoactive agents in a rat tumor model: preliminary results. Contrast Media Mol Imaging. 2010

Schaefer C, Fuhrhop I, Schroeder M, Viezens L, Otten J, Fiedler W, Rüther W, Hansen-Algenstaedt N. Microcirculation of secondary bone tumors in vivo: the impact of minor surgery at a distal site. J Orthop Res. 2010; 28:1515-1521. [PubMed: 20872590]

Shiraishi J, Sugimoto K, Moriyasu F, Kamiyama N, Doi K. Computer-aided diagnosis for the classification of focal liver lesions by use of contrast-enhanced ultrasonography. Med Phys. 2008; 35:1734-1746. [PubMed: 18561648]

Streeter JE, Gessner R, Miles I, Dayton PA. Improving sensitivity in ultrasound molecular imaging by tailoring contrast agent size distribution: in vivo studies. Mol Imaging. 2010; 9:87-95. [PubMed: 20236606]

Sugimoto K, Shiraishi J, Moriyasu F, Ichimura S, Metoki R, Doi K. Analysis of intrahepatic vascular morphological changes of chronic liver disease for assessment of liver fibrosis 17 stages by microflow imaging with contrast-enhanced ultrasound: preliminary experience. Eur Radiol. 2010; 20:2749-2757. [PubMed: 20571803]

Wang Y, Dan HJ, Fan JH, Wen SB. Evaluation of the correlation between colour power Doppler flow imaging and vascular endothelial growth factor in breast cancer. J Int Med Res. 2010; 38:10771083. [PubMed: 20819445]

Wei K, Jayaweera AR, Firoozan S, Linka A, Skyba DM, Kaul S. Quantification of myocardial blood flow with ultrasound-induced destruction of microbubbles administered as a constant venous infusion. Circulation. 1998; 97(5):473-483. [PubMed: 9490243] 


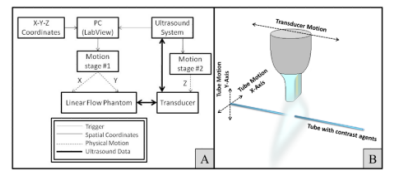

Figure 1.

A: A schematic block-diagram of the phantom generation system. Motion stage 1 and 2 correspond to the Newport and VisualSonics stages respectively. B: A cartoon schematic of the spatial relationships between the physically moving components of the setup. 


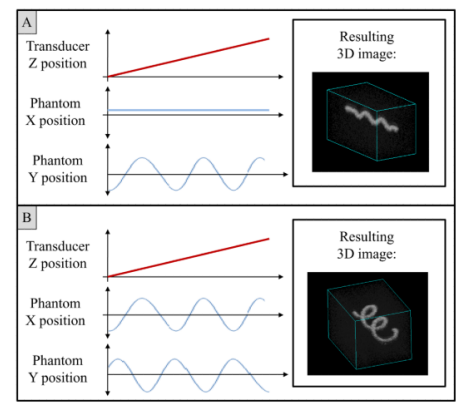

Figure 2.

Two cartoons illustrating the methodology of simulating complex 3D vessel structures by independently controlling the X and Y locations of the vessel's centerline. Blue curves are controlled by the Newport motion stage. Red curves are controlled by the VisualSonics motion stage. A: A sinusoid is generated. B: Two out of phase sinusoids result in the generation of a helix. 


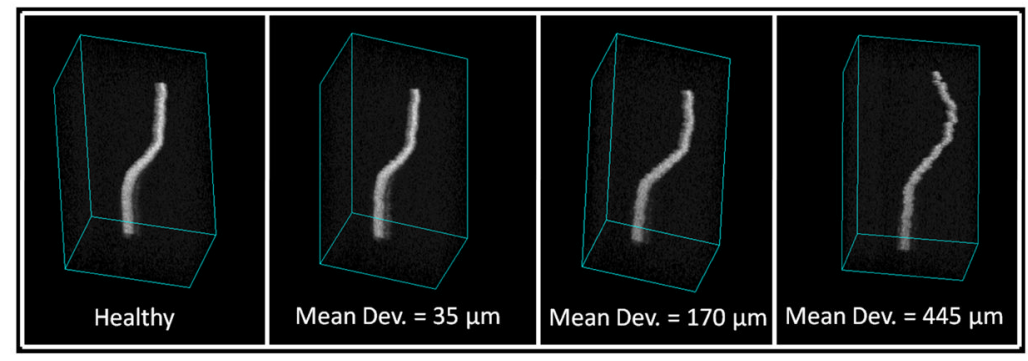

Figure 3.

3D maximum intensity projections of ultrasound data collected of various vessel phantoms. These phantoms represent simulations of a healthy vessel developing high spatial-frequency tortuosity abnormalities which are typical of cancerous vasculature. Mean deviations ("Mean Dev.") from the healthy vessel's centerline range between $35 \mu \mathrm{m}$ and $445 \mu \mathrm{m}$. 


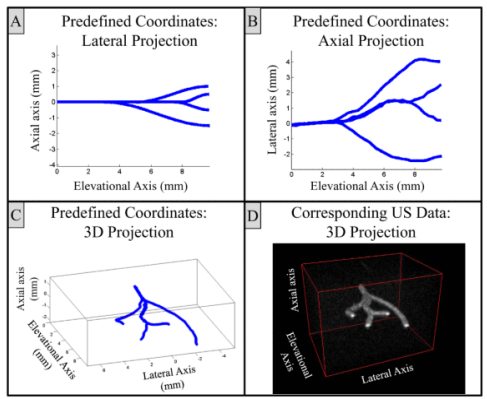

Figure 4.

A and B: Lateral and Axial projections of 3D vessel network coordinate data, respectively. The network was designed so that all bifurcations result in daughter vessels diverging in opposite axial directions, as seen in A. C and D: 3D projections of vessel network coordinate data and ultrasound (US) data respectively. 


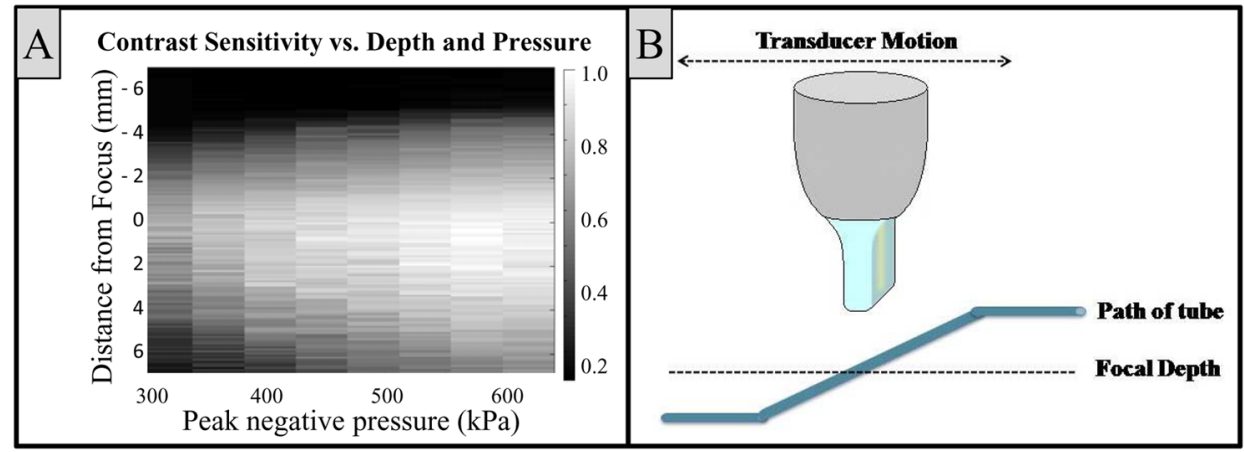

Figure 5.

A: An image displaying the sensitivity of our ultrasound probe to contrast as a function of pulsing pressure. The rows in this image correspond to the distance from the transducer's focus to the tube. The columns in the image represent the discrete pulsing pressures tested between 300 and $650 \mathrm{kPa}$. B: A cartoon illustrating how the tube was moved to simulate a diagonal-sloped "ramp phantom" through 3D space. 